\title{
Revisiting Minimally Invasive Surgery in the Management of Early-Stage Cervical Cancer
}

\author{
Kathryn P. Pennington, MD; Renata R. Urban, MD; and Heidi J. Gray, MD
}

\begin{abstract}
Minimally invasive surgery (MIS) was previously considered an acceptable alternative to open radical hysterectomy in the management of early-stage cervical cancer (ESCC), but adequately powered, high-quality prospective trials evaluating survival outcomes were lacking. Recently, a large randomized phase III trial, the Laparoscopic Approach to Cervical Cancer (LACC) trial, showed that MIS for ESCC is associated with a higher risk of recurrence and death compared with open surgery. We review the LACC trial findings in depth, as well as a recent National Cancer Database analysis using propensity score weighting that supports the LACC trial findings. Additional studies are needed to better understand the mechanisms explaining the worse survival associated with MIS for ESCC. This review discusses considerations for integrating the findings of the LACC trial into clinical practice. Based on the high-quality evidence now available, open radical hysterectomy should be offered as standard of care for stage IA2-IB1 cervical cancer and patients should be guided appropriately to make informed shared decision-making if they still desire MIS.
\end{abstract}

J Natl Compr Canc Netw 2019;17(1):86-90 doi: $10.6004 /$ jnccn.2018.7263
Use of minimally invasive surgery (MIS) in the management of early-stage cervical cancer (ESCC), previously considered an acceptable alternative to open surgery, ${ }^{1}$ was recently challenged by results of the Laparoscopic Approach to Cervical Cancer (LACC) trial, ${ }^{2}$ which demonstrated inferior oncologic outcomes with minimally invasive radical hysterectomy compared with open radical hysterectomy. [Editors Note: The "Principles of Evaluation and Surgical Staging" and accompanying discussion in the NCCN Guidelines for Cervical Cancer, Version 3.2019, published in this issue and on NCCN.org, have been updated to highlight the oncologic risks associated with minimally invasive approaches for radical hysterectomy.] In prior retrospective studies, laparoscopic radical hysterectomy was associated with less blood loss, shorter hospital stay, and fewer complications compared with open radical hysterectomy, ${ }^{3-6}$ but did not seem to have higher recurrence or worse survival rates. ${ }^{6-12}$ However, most of these studies were retrospective single-institution studies lacking adequate power to compare oncologic outcomes. In addition, randomized clinical trials in other cancer types (early-stage endometrial cancer, colorectal cancer) showed similar survival with a minimally invasive approach compared with open surgery. ${ }^{13-16}$

The LACC trial was an international, multicenter, randomized phase III trial of women with stage IA1 (with lymphovascular space invasion), IA2, or IB1 cervical squamous cell carcinoma, adenocarcinoma, or adenosquamous carcinoma of the uterine cervix who were randomized to undergo either open radical hysterectomy (laparotomy) or laparoscopic/robotic radical hysterectomy (type II or III Piver-Rutledge classification). The trial was designed to test for noninferiority of minimally invasive radical hysterectomy (total laparoscopic or robotic radical hysterectomy) compared with open radical hysterectomy, with a planned sample size of 740 patients and 4.5 years of follow-up, to show that MIS was within $7.2 \%$ of the disease-free survival (DFS) rate of the open surgery arm, with at least $90 \%$ power for noninferiority (based on a $97.5 \%$ one-sided CI). Inclusion characteristics of both groups were similar, and there were no significant differ- 
ences in postoperative histopathologic differences between groups with respect to histology, grade, tumor size ( $<2$ vs $\geq 2 \mathrm{~cm}$ ), lymphovascular space invasion, parametrial involvement, or lymph node involvement, although grade and depth of invasion were not reported in approximately $30 \%$ of cases. The MIS group had a higher rate of superficially invasive tumors $(28.5 \%$ vs $21.6 \%$ in the open surgery group). Other pathologic findings were similar: $12 \%$ of women in the MIS group versus $13 \%$ of those in the open group had positive lymph nodes, and parametrial involvement was present in $7 \%$ and $4 \%$ of individuals in each group, respectively. Similar rates of postoperative adjuvant therapy were administered in both groups $(29 \%$ in MIS group, $28 \%$ in open group).

The study was terminated prematurely after $85 \%$ of the planned accrual $(n=631)$ based on recommendations by the Data Safety and Monitoring Committee due to higher rates of recurrence and death in the MIS arm. DFS at 4.5 years was $86.0 \%$ in the MIS group and $96.5 \%$ in the open surgery group (between-group difference, -10.6 percentage points; $95 \% \mathrm{CI},-16.4$ to $-4.7 ; P=.87$ for noninferiority) based on intention-to-treat analysis. Per-protocol analysis revealed similar findings. Minimally invasive radical hysterectomy was associated with a significantly lower DFS than open surgery, with a hazard ratio (HR) for disease recurrence or death from cervical cancer of 3.74 (95\% CI, 1.63-8.58), which persisted after adjustment for age, body mass index (BMI), cancer stage, lymphovascular space invasion, lymph node involvement, and performance status. Overall survival (OS) was also significantly worse in the MIS group (3-year OS, 93.8\% vs $99 \%$ for open surgery; HR for death from any cause, 6.00 ; 95\% CI, 1.77-20.3), as was cancer specific-survival (3-year rate of death from cervical cancer, $4.4 \%$ vs $0.6 \%$ for open surgery; HR, 6.56; 95\% CI, 1.48-29.0). Women in the MIS arm had a significantly higher rate of locoregional recurrence (3-year rate of locoregional recurrence-free survival, $94.3 \%$ vs $98.3 \%$ for MIS; HR for locoregional recurrence, 4.26; 95\% CI, 1.44-12.60).

The unexpected negative results of the LACC trial are staggering: women who received MIS for ESCC were almost 4 times more likely to experience recurrence and were 6.6 times more likely to die of their cancer than those who underwent open surgery. Importantly, although median length of hospital stay was shorter for MIS compared with open surgery (3 vs 5 days), no significant differences were seen in the rates of intraoperative complications, serious adverse events, or long-term morbidities between arms. ${ }^{2}$ Quality of life was assessed using the Functional Assessment of Cancer Therapy-Cervix (FACT-Cx), Short Form-12 (SF-12), M.D. Anderson Symptom Inventory (MDASI), and EuroQol-5D (EQ-5D), and reassuringly, almost all general and disease-specific health-related quality of life scores were similar between groups at all timepoints. ${ }^{17}$ Mobility was decreased in the open surgery group compared with the MIS group at postoperative week 1, but these differences resolved by 6 weeks postoperatively.

Some limitations of the trial included missing data in some patients, a lack of standardization of adjuvant treatment, no central pathology review, and lack of data on patient race and ethnicity. Nonetheless, reported overall inclusion characteristics, postoperative histopathologic findings, and rates of adjuvant treatment were similar between groups.

Findings from a recent population-based study by Melamed et $\mathrm{al}^{18}$ using the National Cancer Database (NCDB) were also consistent with the LACC trial findings of worse oncologic outcomes with MIS. The NCDB analysis included 2,461 patients at Commission on Cancer-accredited US hospitals diagnosed with stage IA2 or IB1 cervical cancer (squamous cell, adenosquamous, or adenocarcinoma) between 2010 and 2013 and treated with radical hysterectomy as primary treatment $(49.8 \%$ minimally invasive). Using extremely robust statistical methods with inverse probability of treatment propensity-score weighting, they found that women treated with MIS had decreased 4-year OS compared with those treated with open surgery (90.9\% vs $94.7 \%$; $P=.002)$, with an HR for death of 1.65 (95\% CI, 1.22-2.22). Although unmeasured confounding or patient selection is always a concern in any nonrandomized study, women in the MIS group would have actually been predicted to have longer survival compared with those in the open surgery group due to their younger age, higher socioeconomic status, and lower tumor grade.

The same authors also performed an interrupted time-series analysis of women who underwent radical hysterectomy for cervical cancer during 2000 to 2010 in the SEER database, and found that the adoption of MIS coincided with an $0.8 \%$ decline in 4 -year relative survival rate per year after 2006 (95\% CI, 0.3-1.4; $P=.01$ for change of trend). A recent subset analysis of the same NCDB population focusing on patients with tumors $\geq 2 \mathrm{~cm}$ also documented a decreased 5-year OS rate for the MIS versus open surgery approach (HR, 2.14; 95\% CI, $1.36-3.38 ; P<.001) .{ }^{19}$

Why did the recent population-based study by Melamed et al $^{18}$ demonstrate inferior oncologic outcomes with MIS, when prior retrospective studies reported similar outcomes compared with open surgery? Prior studies were limited by low power, shorter follow-up, and likely confounding. For example, even in a relatively large matched cohort study by Nam et $\mathrm{al}^{7}$ of 526 women with stage IA2 to IIA cervical cancer who underwent radical hysterectomy (open vs MIS) with a median follow-up of 91 months, those in the MIS group were not found to have a statistically higher risk of death compared with those in the open surgery group. However, the HR for 
death of 1.46 (95\% CI, 0.6-3.4) was of similar magnitude to that reported in the larger NCDB analysis by Melamed et $\mathrm{al}^{18}{ }^{18}$ and the study by Nam et $\mathrm{al}^{7}$ was likely underpowered to detect a difference between groups, because only 23 total deaths were observed.

Further studies are needed to understand why MIS is associated with worse survival in ESCC. Risk of tumor spillage with use of a uterine manipulator has been suggested. Another hypothesis is that $\mathrm{CO}_{2}$ insufflation gas may increase the risk of tumor dissemination. Animal studies exploring the role of $\mathrm{CO}_{2}$ gas in possible tumor spread have been conflicting; some have suggested that $\mathrm{CO}_{2}$ pneumoperitoneum may increase wound and peritoneal metastases compared with laparotomy or gasless laparoscopy, ${ }^{20-22}$ although other studies have failed to demonstrate a difference. ${ }^{23,24}$ An additional consideration is whether the extent of resection differs between minimally invasive and open surgical approaches for radical hysterectomy. One single-institution study suggested that robotic surgery was associated with a higher rate of close surgical margins compared with open surgery, although only 20 cases were robotic and may have been performed during the robotic surgery learning curve. In addition, close surgical margins were not an independent predictor of recurrence in this analysis. ${ }^{25}$ In the LACC trial, no differences were seen between arms in the rates of positive vaginal margins $(2 \%$ in each arm); rates of close surgical margins were not reported.

With the published findings of the LACC trial available, we have an obligation to our patients to practice evidence-based medicine and determine how to appropriately integrate the findings into our clinical practice. Concerns have been raised regarding whether these results should be applied to robotic surgery (vs traditional laparoscopy). Proponents of robotic surgery may argue that the inferior outcomes observed with MIS in the LACC trial cannot be generalized to robotic surgery specifically because of the 289 participants in the MIS arm, only 45 (16\%) had robotic surgery. However, a per-protocol sensitivity analysis of DFS at 4 years revealed nearly identical differences between 4-year DFS rates in the laparoscopy and robotic arms compared with the open arm (laparoscopic: $-10.6 \%$ absolute difference in DFS [95\% CI, -16.4 to -4.7 ]; robotic: $-10.4 \%$ absolute difference DFS [95\% CI, -24.7 to 3.9]). In the study by Melamed et $\mathrm{al}^{18}{ }^{18}$ nearly $80 \%$ of patients in the MIS cohort underwent robotic radical hysterectomy, and exploratory subgroup analyses found that both robotic-assisted and traditional laparoscopic radical hysterectomy were each associated with a higher risk of death compared with open surgery (robotic: HR, 1.61; 95\% CI, 1.18-2.21; laparoscopic: HR, 1.50 ; 95\% CI, 0.97-2.31) (Figure 1). Thus, we feel it would be inappropriate to reassure patients that robotic surgery has similar outcomes compared with open surgery based on the data available.

Even surgeons with extensive experience using MIS to treat ESCC should not be tempted to conclude that the results of the LACC trials are not applicable to them. The LACC trial involved high-volume, experienced cervical cancer surgeons. To ensure proper surgical technique during MIS in the LACC trial, surgeons at participating sites were required to provide 2 unedited videos of a total laparoscopic or robotic radical hysterectomy procedure, independently reviewed by 2 members of the Trial Management Committee, as well as perioperative outcomes of 10 cases. In addition, no sites or individual surgeons performed only the MIS or only the open approach for cervical cancer management.

Importantly, surgeons should not inform patients that they have reviewed their own personal data, and that, in their specific hands, patients can expect equivalent outcomes regardless of surgical approach. As Ramirez et $\mathrm{al}^{2}$ discussed, retrospective studies (whether largescale or surgeon-specific) are often sequential rather than concurrent analyses, in which patients in the open surgery group likely were treated during an earlier time frame, when indications for radical hysterectomy were broader (including patients with stage IB2 disease), recommendations for radiotherapy may not have been clearly defined, or the addition of chemotherapy was not standard practice. In addition, smaller concurrent analyses are likely subject to confounding factors, including significant selection bias. Single-surgeon and even single-institutional analyses would be significantly underpowered to detect the difference between patients having MIS or open surgery.

Whether MIS should be offered to patients with lower-risk ESCC (tumors $<2 \mathrm{~cm}$ ) is uncertain. Neither the LACC trial nor the Melamed et a ${ }^{18}$ study were adequately powered to evaluate oncologic outcomes in patients with tumors $<2 \mathrm{~cm}$. In the LACC trial, $52 \%$ of patients in each trial arm had tumors $<2 \mathrm{~cm}$ : 147 in the open surgery group and 150 in the MIS group; 6 recurrences occurred in patients with tumors $<2 \mathrm{~cm}$. Disease recurrence occurred in 1 patient $(0.68 \%)$ in the open surgery group and $5(3.33 \%)$ in the MIS group. Although the number of recurrences in each group was small, a post hoc subgroup analysis could not distinguish a different impact by surgical approach for smaller versus larger tumors. The NCDB analysis by Melamed et $\mathrm{al}^{18}$ found that the association between MIS and increased all-cause mortality remained across histologic type and tumor sizes (Figure 1). Specifically, for tumor size $<2 \mathrm{~cm}$, the HR for death was 1.46 (95\% CI, 0.703.02), and although the CI crossed 1, the association was consistent with that seen in the primary analysis and in all subanalyses. The authors comment that they were unable 


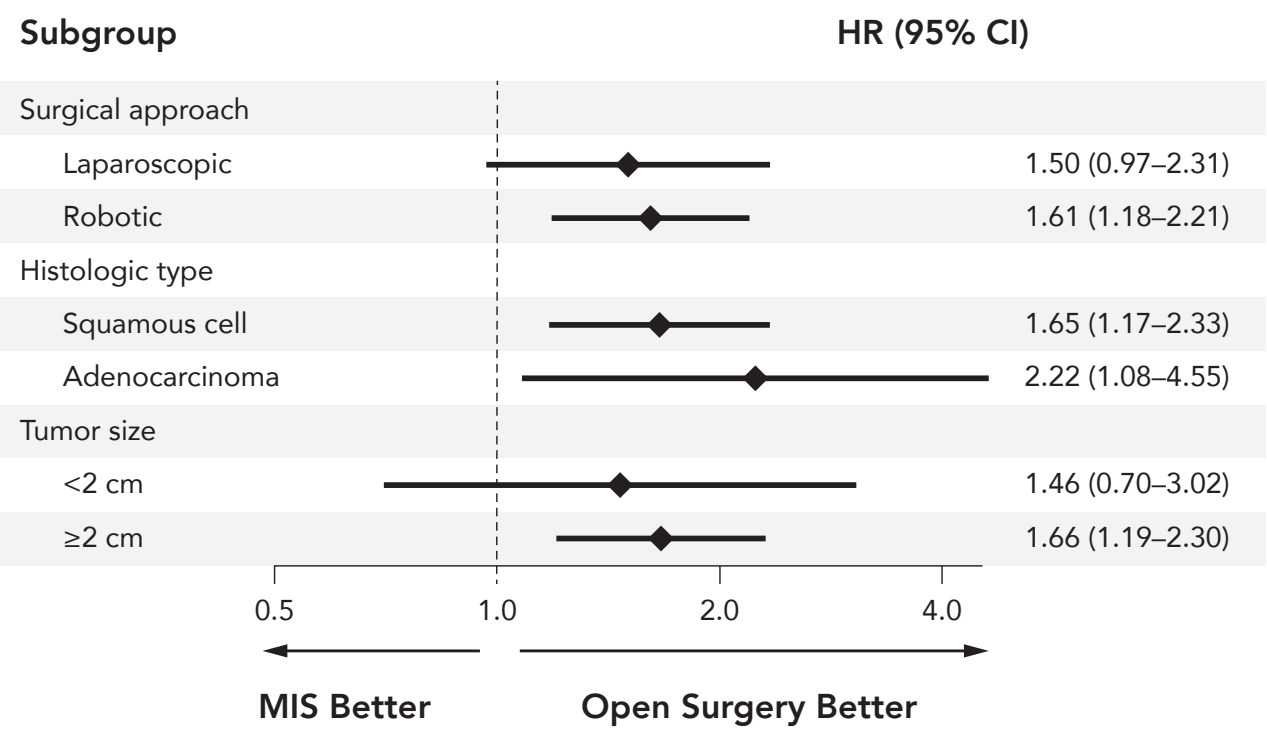

Figure 1. Subgroup analyses by showing the associations between minimally invasive radical hysterectomy and all-cause mortality according to mode of MIS, histologic type, and tumor size.

Abbreviations: HR, hazard ratio; MIS, minimally invasive surgery.

From Melamed A, Margul DJ, Chen L, et al. Survival after minimally invasive radical hysterectomy for early-stage cervical cancer. N Engl J Med 2018;379:1905-1914. Copyright @ Massachusetts Medical Society. Reprinted with permission from Massachusetts Medical Society.

to precisely estimate the association between MIS and mortality in the subgroup of women who had tumors $<2$ $\mathrm{cm}$ because there were few deaths in that subgroup. Based on the available data, we have concerns about offering MIS even to women with tumors $<2 \mathrm{~cm}$, and recommend that women be counseled that it is unknown whether MIS can offer similar oncologic outcomes to open surgery for these smaller lesions.

Although the LACC trial did not find any differences in rates of intraoperative complications, serious adverse events, or long-term morbidities between arms, the potential increased morbidity of open surgery compared with MIS remains relevant. Median BMI of participants in the LACC trial was only $26 \mathrm{~kg} / \mathrm{m}^{2}$ (range, $21-32 \mathrm{~kg} / \mathrm{m}^{2}$ ), which is likely significantly lower than the average BMI for patients with ESCC in the United States. In a recent analysis by Uppal et $\mathrm{al}^{26}$ of 7,180 patients treated with radical hysterectomy for cervical cancer in the National Inpatient Sample from 2012 through 2015 in the United States, $10 \%$ had a BMI of 30.0 to 39.9 , and an additional $5 \%$ had a $B M I \geq 40$. In this study, the overall rate of at least one complication during hospitalization after open surgery was $24.8 \%$ compared with $10 \%$ in MIS cases $(P<.001)$, with a higher risk of infectious and medical complications, but not surgical complications, associated with open surgery. Nonetheless, the potential increased risk of complications likely does not supersede the concern regarding a 6 -fold higher risk of dying associated with MIS for unselected patients. For the much smaller subset of patients who are at significantly high risk of major complications or severe morbidity with open surgery, perhaps primary chemoradiation (which has equivalent oncologic outcomes to open surgery) should be a consideration.

In summary, MIS for ESCC is associated with a higher risk of recurrence and death compared with open surgery. The level I evidence presented by the randomized controlled LACC trial should not be ignored in favor of earlier level II-III data from smaller, retrospective studies when guiding clinical decision-making. Additional studies are needed to better understand the mechanisms explaining these contemporary findings and to determine whether any subgroups of patients could still routinely benefit from MIS, even if they have smaller tumors. It is critical to counsel patients accordingly. Based on the high-level evidence from the LACC trial and the NCDB analysis, open radical hysterectomy should be offered as standard of care for stage IA2-IB1 cervical cancer and patients should be guided appropriately to make informed shared decision-making if they still desire MIS.

Submitted December 9, 2018; accepted for publication December 17, 2018 Disclosures: The authors have disclosed that they have no financial interests, arrangements, affiliations, or commercial interests with the manufacturers of any products discussed in this article or their competitors.

Correspondence: Kathryn P. Pennington, MD, Division of Gynecologic Oncology, Department of Obstetrics and Gynecology, University of Washington, 1959 NE Pacific Street, Box 356460, Seattle, WA 98195-6460. Email: katypenn@u.washington.edu 


\section{References}

1. Koh WJ, Abu-Rustum NR, Bean S, et al. NCCN Clinical Practice Guidelines in Oncology: Cervical Cancer. Version 1.2019. Accessed December 12,2018 . To view the most recent version of these guidelines, visit NCCN.org.

2. Ramirez PT, Frumovitz $M$, Pareja $R$, et al. Minimally invasive versus abdominal radical hysterectomy for cervical cancer. N Engl J Med 2018;379:1895-1904.

3. Piedimonte S, Czuzoj-Shulman N, Gotlieb W, et al. Robotic radical hysterectomy for cervical cancer: a population-based study of adoption and immediate postoperative outcomes in the United States [published online September 5, 2018]. J Minim Invasive Gynecol. doi: 10.1016/j. jmig.2018.08.012

4. Shazly SA, Murad MH, Dowdy SC, et al. Robotic radical hysterectomy in early stage cervical cancer: a systematic review and meta-analysis. Gynecol Oncol 2015;138:457-471.

5. Zhang S, Wang S, Lv A, et al. Laparoscopically assisted radical vaginal hysterectomy for early-stage cervical cancer: a systemic review and meta-analysis. Int J Gynecol Cancer 2016;26:1497-1502.

6. Shah CA, Beck T, Liao JB, et al. Surgical and oncologic outcomes after robotic radical hysterectomy as compared to open radical hysterectomy in the treatment of early cervical cancer. J Gynecol Oncol 2017;28:e82.

7. Nam JH, Park JY, Kim DY, et al. Laparoscopic versus open radical hysterectomy in early-stage cervical cancer: long-term survival outcomes in a matched cohort study. Ann Oncol 2012;23:903-911.

8. Sert BM, Boggess JF, Ahmad S, et al. Robot-assisted versus open radical hysterectomy: a multi-institutional experience for early-stage cervical cancer. Eur J Surg Oncol 2016;42:513-522.

9. Malzoni M, Tinelli R, Cosentino F, et al. Total laparoscopic radical hysterectomy versus abdominal radical hysterectomy with lymphadenectomy in patients with early cervical cancer: our experience. Ann Surg Oncol 2009;16:1316-1323.

10. Bogani G, Cromi A, Uccella S, et al. Laparoscopic versus open abdominal management of cervical cancer: long-term results from a propensity-matched analysis. J Minim Invasive Gynecol 2014;21:857-862.

11. Diver E, Hinchcliff E, Gockley A, et al. Minimally invasive radical hys terectomy for cervical cancer is associated with reduced morbidity and similar survival outcomes compared with laparotomy. J Minim Invasive Gynecol 2017;24:402-406.

12. Mendivil AA, Rettenmaier MA, Abaid LN, et al. Survival rate comparisons amongst cervical cancer patients treated with an open, robotic-assisted or laparoscopic radical hysterectomy: a five year experience. Surg Oncol 2016;25:66-71.

13. Walker JL, Piedmonte MR, Spirtos NM, et al. Recurrence and survival after random assignment to laparoscopy versus laparotomy for comprehensive surgical staging of uterine cancer: Gynecologic Oncology Group LAP2 study. J Clin Oncol 2012;30:695-700.
14. Fleshman J, Sargent DJ, Green E, et al. Laparoscopic colectomy for cancer is not inferior to open surgery based on 5-year data from the COST Study Group trial. Ann Surg 2007;246:655-662; discussion 662-664.

15. Janda M, Gebski V, Davies LC, et al. Effect of total laparoscopic hysterectomy vs total abdominal hysterectomy on disease-free survival among women with stage I endometrial cancer: a randomized clinical trial. JAMA 2017;317:1224-1233.

16. Bonjer $\mathrm{HJ}$, Deijen $\mathrm{CL}$, Abis GA, et al. A randomized trial of laparoscopic versus open surgery for rectal cancer. N Engl J Med 2015;372:13241332.

17. Frumovitz $\mathrm{M}$, Obermair A, Pareja R, et al. Quality of life in early-stage cervical cancer patients undergoing radical hysterectomy via minimally invasive or open surgery: the LACC trial. Presented at the 17th Biennial Meeting of the International Gynecologic Cancer Society; September 14-16, 2018; Kyoto, Japan. Available at: https://journals.Iww.com/ijgc/ Documents/IGCS\%202018\%20Meeting\%20Abstracts,\%20Kyoto.pdf.

18. Melamed A, Margul DJ, Chen L, et al. Survival after minimally invasive radical hysterectomy for early-stage cervical cancer. $\mathrm{N}$ Engl J Med 2018;379:1905-1914.

19. Margul DJ, Yang J, Seagle BL, et al. Outcomes and costs of open, robotic, and laparoscopic radical hysterectomy for stage IB1 cervical cancer [abstract]. J Clin Oncol 2018;36(Suppl):Abstract 5502.

20. Hopkins MP, Dulai RM, Occhino A, et al. The effects of carbon dioxide pneumoperitoneum on seeding of tumor in port sites in a rat model. Am J Obstet Gynecol 1999;181:1329-1333; discussion 1333-1334.

21. Mathew G, Watson DI, Ellis TS, et al. The role of peritoneal immunity and the tumour-bearing state on the development of wound and peritoneal metastases after laparoscopy. Aust N Z J Surg 1999;69:14-18.

22. Volz J, Koster S, Spacek Z, et al. The influence of pneumoperitoneum used in laparoscopic surgery on an intraabdominal tumor growth. Cancer 1999;86:770-774.

23. Lecuru F, Agostini A, Camatte $S$, et al. Impact of pneumoperitoneum on tumor growth. Surg Endosc 2002;16:1170-1174.

24. Mo X, Yang Y, Lai H, et al. Does carbon dioxide pneumoperitoneum enhance wound metastases following laparoscopic abdominal tumor surgery? A meta-analysis of 20 randomized control studies. Tumour Biol 2014;35:7351-7359.

25. McCann GA, Taege SK, Boutsicaris CE, et al. The impact of close surgical margins after radical hysterectomy for early-stage cervical cancer Gynecol Oncol 2013;128:44-48.

26. Uppal S, Rebecca Liu J, Reynolds RK, et al. Trends and comparative effectiveness of inpatient radical hysterectomy for cervical cancer in the United States (2012-2015) [published online November 10, 2018]. Gynecol Oncol. doi: 10.1016/j.ygyno.2018.09.027 